PROCEEDINGS OF THE

AMERICAN MATHEMATICAL SOCIETY

Volume 140, Number 12, December 2012, Pages 4369-4380

S 0002-9939(2012)11283-3

Article electronically published on April 19, 2012

\title{
EXPECTED LENGTH OF A PRODUCT OF RANDOM REFLECTIONS
}

\author{
JONAS SJÖSTRAND
}

(Communicated by Jim Haglund)

\begin{abstract}
We present a simple formula for the expected number of inversions in a permutation of size $n$ obtained by applying $t$ random (not necessarily adjacent) transpositions to the identity permutation. More generally, for any finite irreducible Coxeter group belonging to one of the infinite families (type A, B, D, and I), an exact expression is obtained for the expected length of a product of $t$ random reflections.
\end{abstract}

\section{INTRODUCTION}

In 2000, Eriksson et al. 6] studied the expected number of inversions after $t$ random adjacent transpositions applied to the identity permutation. Their main motivation came from the area of molecular evolution where a genome (modelled as a permutation) undergoes random mutations (modelled as adjacent transpositions). Since then several people have analysed this and similar problems, motivated both by biology and pure mathematics.

In 2005, Eriksen [3] improved the results from [6] and obtained an exact formula for the expected number of inversions. More recently, in 2010, Bousquet-Mélou 2 gave a completely different exact expression for the same thing! In 2002, Troili [8] generalized the question to other groups than the symmetric group, and she gave corresponding exact formulas for the dihedral groups $I_{2}(m)$.

In 2004, Eriksen and Hultman 4 modelled the genome rearrangement problem closer to reality by considering any random transpositions (and not just adjacent ones) as mutations. Instead of the number of inversions they computed the expected absolute length of the resulting permutation, i.e. the minimal number of transpositions whose product is the permutation. In a sequel [5] they generalized their results to a class of complex reflection groups.

For an exposé of the research on more general aspects of Markov chains generated by random (adjacent) transpositions, such as mixing times, we refer to BousquetMélou [2].

In the present paper, we will generate permutations in the same way as Eriksen and Hultman, i.e. by random transpositions, but we will measure them by the number of inversions as in the original papers. We will also generalize the framework to all finite Coxeter groups and present exact expectancy formulas for the groups

Received by the editors November 24, 2010 and, in revised form, May 31, 2011.

2010 Mathematics Subject Classification. Primary 60J10; Secondary 05A05.

Key words and phrases. Permutation, transposition, inversion, Coxeter group, reflection, absolute length.

(C)2012 American Mathematical Society Reverts to public domain 28 years from publication 
$A_{n}, B_{n}, D_{n}$ and $I_{2}(m)$. In Coxeter group lingo, we compute the expected length of a product of $t$ random reflections.

The paper is organized as follows. After a section with some basic definitions, we review the previously known results mentioned above so that the reader gets a feeling for what kind of expressions may appear for the expected (absolute) length and also what type of combinatorics is going on behind the formulas. Next, in section 4 we loosen up by computing the expected length and absolute length of a product of random reflections or simple reflections in the dihedral groups $I_{2}(m)$. After this relatively simple task, in section 5 we are ready to state and prove our main results: formulas for the expected length of a product of random reflections in the Weyl groups of types A, B, and D. Finally, in section 6 we summarize the current state of affairs and reveal some of our ideas for future research.

\section{DEFINITIONS AND NOTATION}

We will follow the notation from the book by Björner and Brenti [1, where the definitions of the basic concepts may also be found. For the reader's convenience we have included the most relevant definitions below.

A Coxeter group is a group $W$ with a presentation of the form

$$
\left.\langle S|\left(s s^{\prime}\right)^{m\left(s, s^{\prime}\right)}=\text { id for all } s, s^{\prime} \in S \text { with } m\left(s, s^{\prime}\right) \neq \infty\right\rangle,
$$

where $m$ is a function from $S$ to $\{1,2, \ldots, \infty\}$ such that $m\left(s, s^{\prime}\right)=m\left(s^{\prime}, s\right)$ for all $s, s^{\prime} \in S$ and $m\left(s, s^{\prime}\right)=1$ if and only if $s=s^{\prime}$. The elements of $S$ (which we identify with their image in $W$ through the presentation) are called generators or simple reflections and the pair $(W, S)$ is called a Coxeter system. The group elements that are conjugate to some simple reflection are called reflections, and the set of reflections is usually denoted by $T$.

By definition, any group element $w \in W$ is a (finite) product of simple reflections, and the minimal $\ell$ such that $w$ is a product of $\ell$ simple reflections is called the length of $w$ and is denoted by $\ell(w)$. Similarly, the minimal $\ell^{\prime}$ such that $w$ is a product of $\ell^{\prime}$ reflections is called the absolute length of $w$ and is denoted by $\ell^{\prime}(w)$.

Now, let us make a very general definition.

Definition 2.1. Given a (multiplicative) monoid $M$, a nonempty finite subset $R \subseteq M$, a "length" function $\varphi$ from $M$ to $\mathbb{N}=\{0,1, \ldots\}$, and an integer $t \geq 0$, we define

$$
E_{R, \varphi}^{M}(t):=\frac{1}{|R|^{t}} \sum_{r_{1}, \ldots, r_{t} \in R} \varphi\left(r_{1} \cdots r_{t}\right)
$$

to be the expected "length" of a product of $t$ random elements, chosen independently and uniformly from $R$.

As a special case, for a Coxeter system $(W, S)$ with $S$ finite, $E_{S, \ell}^{W}(t)$ denotes the expected length of a product of $t$ simple reflections. Similarly, if $W$ is finite, $E_{T, \ell^{\prime}}^{W}(t)$ denotes the expected absolute length of a product of $t$ reflections.

As mentioned in the introduction, exact formulas for $E_{S, \ell}^{W}(t)$ have been found for the symmetric groups $(W, S)=A_{n}$ by Eriksen [3] and for the dihedral groups $(W, S)=I_{2}(m)$ by Troili $[8]$. Exact formulas for $E_{T, \ell^{\prime}}^{W}(t)$ have been found by Eriksen and Hultman, first for the symmetric groups [4] and later for the complex reflection groups $G(r, 1, n)$, where the hyperoctahedral groups $B_{n}$ are included. 
In the present paper we will compute $E_{T, \ell}^{W}$ for the three infinite families of finite irreducible Coxeter systems $A_{n}, B_{n}, D_{n}$, and $I_{2}(m)$.

\section{KNOWN RESULTS}

To get a flavour of the kind of expressions that may appear, we will begin by presenting the already known results and discussing briefly what methods were used to obtain them before we reveal our own results in the next section.

Theorem 3.1 (Eriksen (2005), Bousquet-Mélou (2010)). Let $n \geq 1$ and $t \geq 0$. The expected number of inversions after $t$ random adjacent transpositions applied to the identity permutation in $A_{n}$ is

$$
E_{S, \ell}^{A_{n}}(t)=\sum_{r=1}^{t} \frac{1}{n^{r}}\left(\begin{array}{l}
t \\
r
\end{array}\right) \sum_{s=1}^{r}\left(\begin{array}{l}
r-1 \\
s-1
\end{array}\right)(-1)^{r-s} 4^{r-s} g_{s, n},
$$

where

$$
g_{s, n}=\sum_{l=0}^{n} \sum_{k \in \mathbb{N}}(-1)^{k}(n-2 l)\left(\begin{array}{c}
2\lceil s / 2\rceil-1 \\
\lceil s / 2\rceil+l+k(n+1)
\end{array}\right) \sum_{j \in \mathbb{Z}}(-1)^{j}\left(\begin{array}{c}
2\lceil s / 2\rceil \\
\lceil s / 2\rceil+j(n+1)
\end{array}\right) .
$$

An alternative formula is

$E_{S, \ell}^{A_{n}}(t)=\frac{n(n+1)}{4}-\frac{1}{8(n+1)^{2}} \sum_{k, j=0}^{n} \frac{\left(\cos \alpha_{j}+\cos \alpha_{k}\right)^{2}}{\sin ^{2} \alpha_{j} \sin ^{2} \alpha_{k}}\left(1-\frac{4}{n}\left(1-\cos \alpha_{j} \cos \alpha_{k}\right)\right)^{t}$,

where $\alpha_{k}=(2 k+1) \pi /(2 n+2)$.

The proof of the first formula, due to Eriksen, is built on a discrete heat flow process in a two-dimensional lattice. His expression has a combinatorial interpretation in terms of lattice walks. The second formula, due to Bousquet-Mélou, was obtained by attacking the same heat flow process by generating functions and the kernel method. It has the advantage that the dependence on $t$ is very explicit, which makes it possible to analyze what happens in the limit when $n$ and $t$ tend to infinity at the same time in various ways.

Theorem 3.2 (Troili, 2002). Let $m \in\{2,3, \ldots, \infty\}$ and let $t \geq 0$. Then the expected length of a product of $t$ random simple reflections in the dihedral group $I_{2}(m)$ is

$$
E_{S, \ell}^{I_{2}(m)}(t)=\sum_{j=0}^{\left\lfloor\frac{t-1}{2}\right\rfloor} \frac{1}{4^{j}}\left[\left(\begin{array}{c}
2 j \\
j
\end{array}\right)+2 \sum_{k \geq 1}\left(\begin{array}{c}
2 j \\
j-k m
\end{array}\right)\right]-\Sigma
$$

where

$$
\Sigma= \begin{cases}\sum_{j=1}^{\left\lfloor\frac{t-1}{2}\right\rfloor} \frac{2}{4^{j}} \sum_{k \geq 0}\left(\begin{array}{c}
2 j \\
j-\frac{m}{2}-k m
\end{array}\right) & \text { if } m \text { is even, } \\
\sum_{j=1}^{\left\lfloor\frac{t}{2}\right\rfloor} \frac{2}{4^{j}} \sum_{k \geq 0}\left(\frac{2 j-1-m}{2}-k m\right) & \text { if } m \text { is odd. }\end{cases}
$$

Here, binomial coefficients $\left(\begin{array}{l}a \\ b\end{array}\right)$ with $b$ negative $($ or $b=-\infty)$ vanish by definition.

Troili's proof is built on the observation that

$$
E_{S, \ell}^{I_{2}(m)}(t)=\sum_{r=0}^{t-1}\left[\operatorname{Prob}\left(\ell\left(\pi^{(r)}\right)=0\right)-\operatorname{Prob}\left(\ell\left(\pi^{(r)}\right)=m\right)\right],
$$

where $\pi^{(r)}$ is a product of $r$ random reflections in $I_{2}(m)$. 
Theorem 3.3 (Eriksen, Hultman, 2005). Let $r, n$ be positive integers not both equal to one. Then the expected absolute length of a product of $t$ random reflections in the complex reflection group $G(r, 1, n)$ is

$$
\begin{aligned}
E_{T, \ell^{\prime}}^{G(r, 1, n)}(t) & \\
=n-\frac{1}{r} \sum_{k=1}^{n} \frac{1}{k}+ & \frac{1}{r} \sum_{p=1}^{n-1} \sum_{q=1}^{\min (p, n-p)} a_{p q}\left(\frac{r\left(\left(\begin{array}{c}
p \\
2
\end{array}\right)+\left(\begin{array}{c}
q-1 \\
2
\end{array}\right)-\left(\begin{array}{c}
n-p-q+2 \\
2
\end{array}\right)+n\right)-n}{r\left(\begin{array}{c}
n+1 \\
2
\end{array}\right)-n}\right)^{t} \\
& +\frac{r-1}{r} \sum_{p=0}^{n-1} \sum_{q=1}^{n-p} b_{p q}\left(\frac{r\left(\left(\begin{array}{c}
p \\
2
\end{array}\right)+\left(\begin{array}{c}
q \\
2
\end{array}\right)-\left(\begin{array}{c}
n-p-q+1 \\
2
\end{array}\right)+p\right)-n}{r\left(\begin{array}{c}
n+1 \\
2
\end{array}\right)-n}\right)^{t},
\end{aligned}
$$

where

and

$$
a_{p q}=(-1)^{n-p-q+1} \frac{(p-q+1)^{2}}{(n-q+1)^{2}(n-p)}\left(\begin{array}{c}
n \\
p
\end{array}\right)\left(\begin{array}{c}
n-p-1 \\
q-1
\end{array}\right)
$$

$$
b_{p q}=\frac{(-1)^{n-p-q+1}}{n-p}\left(\begin{array}{c}
n \\
p
\end{array}\right)\left(\begin{array}{c}
n-p-1 \\
q-1
\end{array}\right) .
$$

Note that $A_{n-1} \simeq G(1,1, n)$ and $B_{n} \simeq G(2,1, n)$.

The proof of Eriksen and Hultman's formula involves group representations and characters. To see why this is natural, think of the symmetric group $A_{n-1}$, where the absolute length of a permutation is $n$ minus the number of cycles in the cycle representation of the permutation. Applying a random transposition either splits a cycle or merges two cycles, and it is easy to see that during this process we only have to keep track of the cycle type of the permutation, i.e. its conjugacy class.

Using the same technique as Eriksen and Hultman, recently Andreas Jönsson [7, Th. 4.2] was able to obtain the following formula for the fixed-point distance $\operatorname{fp}(\pi)$ defined as $n$ minus the number of fixed points (i.e. cycles of length 1) of $\pi \in A_{n-1}$.

Theorem 3.4 (Jönsson 2009). Let $n \geq 2$ and let $t \geq 0$. Then the expected fixedpoint distance of a product of $t$ random reflections in the symmetric group $A_{n-1}$ is

$$
E_{T, \mathrm{fp}}^{A_{n-1}}(t)=(n-1)-(n-1)\left(1-\frac{2}{n}\right)^{t} .
$$

The reason that the same technique works in this case is that the fixed-point distance, like the absolute length, is a class function; i.e. it is constant on conjugacy classes. Indeed, Eriksen and Hultman's approach would produce some kind of formula for the expected value of any class function after $t$ random transpositions, at least in principle.

\section{The DIHEDRAL GROUPS}

As we have seen, computing $E_{S, \ell}^{I_{2}(m)}(t)$ for the dihedral groups leads to a rather cumbersome expression in Theorem 3.2. In this section, we will see that much nicer formulas exist for $E_{T, \ell}^{I_{2}(m)}(t), E_{S, \ell^{\prime}}^{I_{2}(m)}(t)$, and $E_{T, \ell^{\prime}}^{I_{2}(m)}(t)$.

Theorem 4.1. For $m \geq 2$ and $t \geq 1$, the expected length $E_{T, \ell}^{I_{2}(m)}(t)$ of a product of $t$ random reflections in the dihedral group $I_{2}(m)$ is $m / 2$ (for all positive $t$ ) if $m$ is even and $\frac{m}{2}-\frac{(-1)^{t}}{2 m}$ if $m$ is odd. 
Proof. Note that in $I_{2}(m)$ the set $T$ of reflections is precisely the set of elements with odd length. Thus, multiplying any element in $T$ by a uniformly chosen random reflection yields a uniformly chosen random element in $I_{2}(m) \backslash T$, and, similarly, multiplying any element in $I_{2}(m) \backslash T$ by a uniformly chosen random reflection yields a uniformly chosen random element in $T$. A simple calculation reveals that the average length of an element in $T$ is $m / 2$ if $m$ is even and $\frac{m}{2}+\frac{1}{2 m}$ if $m$ is odd and that the average length of an element in $I_{2}(m) \backslash T$ is $m / 2$ if $m$ is even and $\frac{m}{2}-\frac{1}{2 m}$ if $m$ is odd.

Theorem 4.2. For $m \in\{2,3, \ldots, \infty\}$ and $t \geq 0$, the expected absolute length $E_{S, \ell^{\prime}}^{I_{2}(m)}(t)$ of a product of $t$ random simple reflections in the dihedral group $I_{2}(m)$ is 1 if $t$ is odd and

$$
2-\frac{1}{2^{t-1}} \sum_{|k| \leq\lfloor t / 2 m\rfloor}\left(\begin{array}{c}
t \\
\frac{t}{2}-k m
\end{array}\right)
$$

if $t$ is even. (If $m=\infty$, the sum contains only one term.)

Proof. Clearly, in a dihedral group, the absolute length of an element $w$ is 1 if $\ell(w)$ is odd and 2 if $\ell(w)$ is even, unless $w=$ id. The probability that a product of an even number $t$ of simple reflections is the identity element is precisely the sum above divided by $2^{t}$.

Theorem 4.3. For $m \geq 2$ and $t \geq 1$, the expected absolute length $E_{T, \ell^{\prime}}^{I_{2}(m)}(t)$ of a product of $t$ random reflections in the dihedral group $I_{2}(m)$ is 1 if $t$ is odd and $2-\frac{2}{m}$ if $t$ is even.

Proof. The theorem follows from the same reasoning as in the proof of Theorem 4.1 and the observation that the absolute length of any element in $T$ is 1 and the average absolute length of an element in $I_{2}(m) \backslash T$ is $2-\frac{2}{m}$.

\section{The Weyl groups of types A, B And D}

In this section we will obtain exact expressions for $E_{T, \ell}^{W}(t)$ when $W$ is a Weyl group of types A, B, and D. The formulas and their proofs turn out to be quite similar for these three types, but the symmetric groups happen to be a bit less technical to deal with, so we start with those.

\subsection{The symmetric groups.}

Theorem 5.1. For $n \geq 2$ and $t \geq 0$, let $\pi^{(t)} \in A_{n-1}$ be the random permutation obtained by applying $t$ random transpositions to the identity permutation. Then the following hold.

(a) The expected number of inversions in $\pi^{(t)}$ is

$$
E_{T, \ell}^{A_{n-1}}(t)=\frac{n(n-1)}{4}-\frac{(n+1)(n-1)}{6}\left(1-\frac{2}{n-1}\right)^{t}-\frac{(n-1)(n-2)}{12}\left(1-\frac{4}{n-1}\right)^{t} .
$$

(b) For $1 \leq i<j \leq n$, the probability that $\pi_{i}^{(t)}>\pi_{j}^{(t)}$ is an inversion is

$$
\frac{1}{2}-\frac{j-i}{n}\left(1-\frac{2}{n-1}\right)^{t}+\left(\frac{j-i}{n}-\frac{1}{2}\right)\left(1-\frac{4}{n-1}\right)^{t} .
$$


Proof. Part (a) of the theorem follows from part (b) by the simple observation that $\sum_{1 \leq i<j \leq n}(j-i)=n\left(n^{2}-1\right) / 6$.

It remains to prove part (b). For every $1 \leq i, j \leq n$ and $t \geq 0$, let

$$
p_{i, j}^{(t)}:=\operatorname{Prob}\left(\pi_{i}^{(t)}<\pi_{j}^{(t)}\right) \quad \text { and } \quad u_{i, j}^{(t)}:=\left(\begin{array}{l}
n \\
2
\end{array}\right)^{t} p_{i, j}^{(t)} .
$$

Clearly, $u_{i, i}^{(t)}=0$ and for $i \neq j$,

$$
u_{i, j}^{(t+1)}=\left(\begin{array}{c}
n \\
2
\end{array}\right) u_{i, j}^{(t)}+\left(u_{j, i}^{(t)}-u_{i, j}^{(t)}\right)+\sum_{i^{\prime} \neq j}\left(u_{i^{\prime}, j}^{(t)}-u_{i, j}^{(t)}\right)+\sum_{j^{\prime} \neq i}\left(u_{i, j^{\prime}}^{(t)}-u_{i, j}^{(t)}\right) .
$$

Now, define $v_{i, j}^{(t)}:=u_{i, j}^{(t)}-u_{j, i}^{(t)}$ for $1 \leq i, j \leq n$. The above recurrence for $u$ translates to the following recurrence for $v$ :

$$
v_{i, j}^{(t+1)}=\left(\begin{array}{c}
n \\
2
\end{array}\right) v_{i, j}^{(t)}-2 v_{i, j}^{(t)}+\sum_{i^{\prime} \neq j}\left(v_{i^{\prime}, j}^{(t)}-v_{i, j}^{(t)}\right)+\sum_{j^{\prime} \neq i}\left(v_{i, j^{\prime}}^{(t)}-v_{i, j}^{(t)}\right) .
$$

Using that $v_{i, i}^{(t)}=0$ for any $i$, we can write the recurrence in a more elegant way:

$$
v_{i, j}^{(t+1)}=\frac{n(n-5)}{2} v_{i, j}^{(t)}+\sum_{i^{\prime}=1}^{n} v_{i^{\prime}, j}^{(t)}+\sum_{j^{\prime}=1}^{n} v_{i, j^{\prime}}^{(t)} .
$$

Next, we will express the above recurrence in terms of linear operators. Let $\mathcal{A}_{n}$ denote the vector space over $\mathbb{R}$ of all real antisymmetric $n \times n$ matrices, and define the linear operator $Q$ on $\mathcal{A}_{n}$ as follows. For any $v \in \mathcal{A}_{n}$ and for any $1 \leq i, j \leq n$, let each entry in $Q v$ be its row sum plus its column sum in $v$ or, formally,

$$
(Q v)_{i, j}=\sum_{i^{\prime}=1}^{n} v_{i^{\prime}, j}+\sum_{j^{\prime}=1}^{n} v_{i, j^{\prime}}
$$

Now, the recurrence relation (5.2) can be written as

$$
v^{(t+1)}=(Q+x I) v^{(t)},
$$

where $I$ is the identity operator on $\mathcal{A}_{n}$ and $x=\frac{n(n-5)}{2}$. The starting point $v^{(0)}$ is the $n \times n$ matrix with zeroes on the main diagonal, ones above it and minus ones below it. From the simple but crucial observation that $Q^{2}=n Q$ we deduce that

$$
v^{(t)}=(Q+x I)^{t} v^{(0)}=\left[(n+x)^{t} \frac{Q}{n}+x^{t}\left(I-\frac{Q}{n}\right)\right] v^{(0)} .
$$

Since $\left(Q v^{(0)}\right)_{i, j}=2(j-i)$ we obtain

$$
v_{i, j}^{(t)}=\frac{2(j-i)}{n}(n+x)^{t}+\left(1-\frac{2(j-i)}{n}\right) x^{t}
$$

if $j>i$. Dividing this by $\left(\begin{array}{l}n \\ 2\end{array}\right)^{t}$ yields

$$
1-2 p_{j, i}^{t}=p_{i, j}^{t}-p_{j, i}^{t}=\frac{2(j-i)}{n}\left(1-\frac{2}{n-1}\right)^{t}+\left(1-\frac{2(j-i)}{n}\right)\left(1-\frac{4}{n-1}\right)^{t},
$$

which proves part (b) of the theorem. 
A striking feature of the second part of Theorem 5.1 is that, for a fixed $t$, the probability that $i<j$ is an inversion depends only on the difference $j-i$. This fact is not evident from the definition of the random process, but nevertheless it can be proved without invoking Theorem 5.1.

Proposition 5.2. Fix $n \geq 2$ and $t \geq 0$, and let $\pi \in A_{n-1}$ be a product of $t$ random transpositions. Then, the probability that $\pi_{i}>\pi_{j}$ is an inversion (where $1 \leq i<j \leq n)$ is only dependent on $j-i$.

Proof. Let $\pi^{+k}$ be the permutation obtained by adding $k$ "modulo $n$ " to all entries of $\pi$, i.e.

$$
\pi_{i}^{+k}= \begin{cases}\pi_{i}+k & \text { if } \pi_{i}+k \leq n \\ \pi_{i}+k-n & \text { if } \pi_{i}+k>n\end{cases}
$$

Clearly, for any $1 \leq i<j \leq n$, we have

$$
\operatorname{Prob}\left(\pi_{i}>\pi_{j}\right)=\operatorname{Prob}\left(\pi_{i}^{+k}>\pi_{j}^{+k}\right)+\operatorname{Prob}\left(\pi_{i}>n-k\right)-\operatorname{Prob}\left(\pi_{j}>n-k\right) .
$$

Now, fix $i, j, k$ with $1 \leq i<j<j+k \leq n$. It suffices to prove that

$$
\operatorname{Prob}\left(\pi_{i+k}>\pi_{j+k}\right)=\operatorname{Prob}\left(\pi_{i}>\pi_{j}\right) .
$$

By symmetry of the random process, we have $\operatorname{Prob}\left(\pi_{i}>n-k\right)=\operatorname{Prob}\left(\pi_{j}>\right.$ $n-k$ ) and hence $\operatorname{Prob}\left(\pi_{i}>\pi_{j}\right)=\operatorname{Prob}\left(\pi_{i}^{+k}>\pi_{j}^{+k}\right)$. Equation (5.3) now follows from the observation that $\operatorname{Prob}\left(\pi_{i+k}>\pi_{j+k}\right)=\operatorname{Prob}\left(\pi_{i}^{+k}>\pi_{j}^{+k}\right)$.

5.2. A definition and a lemma. The situation becomes a little more delicate for Weyl groups of types B and D, but in a way that is very similar for these types. To be economical we gather the bit of reasoning that is common for types $\mathrm{B}$ and $\mathrm{D}$ in a definition and a lemma.

Definition 5.3. For any positive integer $n$, let $\mathcal{I}_{n}:=\{(i, j): i, j \in[-n, n] \backslash$ $\{0\},|i| \neq|j|\}$.

Lemma 5.4. Let $n$ be a positive integer and $x$ a real number. For every integer $t \geq 0$, define a function $v^{(t)}: \mathcal{I}_{n} \rightarrow \mathbb{R}$ by the recurrence relation

$$
v_{i, j}^{(t+1)}=x v_{i, j}^{(t)}+\sum_{\left|i^{\prime}\right| \neq|j|} v_{i^{\prime}, j}^{(t)}+\sum_{\left|j^{\prime}\right| \neq|i|} v_{i, j^{\prime}}^{(t)}
$$

together with the initial condition $v_{i, j}^{(0)}=\operatorname{sgn}(j-i)$. Then these functions are given by

$v_{i, j}^{(t)}=\frac{j-i-\operatorname{sgn} j+\operatorname{sgn} i}{n-1}(2 n-2+x)^{t}+\left(\operatorname{sgn}(j-i)-\frac{j-i-\operatorname{sgn} j+\operatorname{sgn} i}{n-1}\right) x^{t}$.

Proof. Let $\mathcal{D}_{n}$ denote the real vector space of all functions $v: \mathcal{I}_{n} \rightarrow \mathbb{R}$ such that $v_{j, i}=-v_{i, j}$ and $v_{-j,-i}=v_{i, j}$ for any $(i, j) \in \mathcal{I}_{n}$. (We write $v_{i, j}$ instead of the more common $v(i, j)$ since we may view $\mathcal{D}_{n}$ as the space of $2 n \times 2 n$ matrices that are antisymmetric about the main diagonal and symmetric about the antidiagonal if we disregard the entries on the diagonals.)

Let $Q$ be the linear operator on $\mathcal{D}_{n}$ defined by

$$
(Q v)_{i, j}=\sum_{\left|i^{\prime}\right| \neq|j|} v_{i^{\prime}, j}+\sum_{\left|j^{\prime}\right| \neq|i|} v_{i, j^{\prime}}
$$

for any $v \in \mathcal{D}_{n}$ and $(i, j) \in \mathcal{I}_{n}$, 
We have

$$
\begin{aligned}
& \left(Q^{2} v\right)_{i, j}=\sum_{\left|i^{\prime}\right| \neq|j|}(Q v)_{i^{\prime}, j}+\sum_{\left|j^{\prime}\right| \neq|i|}(Q v)_{i, j^{\prime}} \\
& =\sum_{\left|i^{\prime}\right| \neq|j|}\left(\sum_{\left|i^{\prime \prime}\right| \neq|j|} v_{i^{\prime \prime}, j}+\sum_{\left|j^{\prime \prime}\right| \neq\left|i^{\prime}\right|} v_{i^{\prime}, j^{\prime \prime}}\right)+\sum_{\left|j^{\prime}\right| \neq|i|}\left(\sum_{\left|i^{\prime \prime}\right| \neq\left|j^{\prime}\right|} v_{i^{\prime \prime}, j^{\prime}}+\sum_{\left|j^{\prime \prime}\right| \neq|i|} v_{i, j^{\prime \prime}}\right) \\
& =(2 n-2) \sum_{\left|i^{\prime \prime}\right| \neq|j|} v_{i^{\prime \prime}, j}+\sum_{\left(j^{\prime \prime}, i^{\prime}\right) \in \mathcal{I}_{n}} v_{i^{\prime}, j^{\prime \prime}}-\sum_{\left|j^{\prime \prime}\right| \neq|j|}\left(v_{j, j^{\prime \prime}}+v_{-j, j^{\prime \prime}}\right) \\
& \quad+\sum_{\left(i^{\prime \prime}, j^{\prime}\right) \in \mathcal{I}_{n}} v_{i^{\prime \prime}, j^{\prime}}-\sum_{\left|i^{\prime \prime}\right| \neq|i|}\left(v_{i^{\prime \prime}, i}+v_{i^{\prime \prime},-i}\right)+(2 n-2) \sum_{\left|j^{\prime \prime}\right| \neq|i|} v_{i, j^{\prime \prime}}
\end{aligned}
$$

(now using the symmetry and antisymmetry properties)

$$
\begin{aligned}
& =(2 n-2)\left(\sum_{\left|i^{\prime \prime}\right| \neq|j|} v_{i^{\prime \prime}, j}+\sum_{\left|j^{\prime \prime}\right| \neq|i|} v_{i, j^{\prime \prime}}\right) \\
& =(2 n-2)(Q v)_{i, j} .
\end{aligned}
$$

Thus, we have shown that $Q^{2}=(2 n-2) Q$, which means that

$$
(Q+x I)^{t} v^{(0)}=\left[(2 n-2+x)^{t} \frac{Q}{2 n-2}+x^{t}\left(I-\frac{Q}{2 n-2}\right)\right] v^{(0)},
$$

where $I$ is the identity operator on $\mathcal{D}_{n}$. The lemma now follows from the observation that

$$
\left(Q v^{(0)}\right)_{i, j}=\sum_{\left|i^{\prime}\right| \neq|j|} \operatorname{sgn}\left(j-i^{\prime}\right)+\sum_{\left|j^{\prime}\right| \neq|i|} \operatorname{sgn}\left(j^{\prime}-i\right)=2(j-i-\operatorname{sgn} j+\operatorname{sgn} i) .
$$

5.3. The hyperoctahedral groups. Recall that $B_{n}$ is isomorphic to the subgroup of permutations $\pi$ of the set $[-n, n] \backslash\{0\}$ such that $\pi_{-i}=-\pi_{i}$ for all $1 \leq i \leq n$ (see e.g. 1, Ch. 8]). In this representation, the set of reflections is

$$
\{(i, j)(-i,-j): 1 \leq|i|<j \leq n\} \cup\{(i,-i): 1 \leq i \leq n\}
$$

in cycle notation. The length of $\pi$ equals its number of $B$-inversions, which are pairs $(i, j)$ with $i, j \in[-n, n] \backslash\{0\}$ and $j \geq|i|$ such that $\pi_{i}>\pi_{j}$.

Theorem 5.5. For $n \geq 1$ and $t \geq 0$, let $\pi^{(t)}$ be a product of $t$ random reflections in $B_{n}$. Then the following hold.

(a) The expected length of $\pi^{(t)}$ is

$$
E_{T, \ell}^{B_{n}}(t)=\frac{n^{2}}{2}-\frac{n(n+1)}{3}\left(1-\frac{2}{n}\right)^{t}-\frac{n(n-2)}{6}\left(1-\frac{4}{n}+\frac{2}{n^{2}}\right)^{t} .
$$

(b) If $i, j \in[-n, n] \backslash\{0\}$ and $j>|i|$, the probability that $\pi_{i}^{(t)}>\pi_{j}^{(t)}$ is

$$
\frac{1}{2}-\frac{j-i-1+\operatorname{sgn} i}{2(n-1)}\left(1-\frac{2}{n}\right)^{t}+\left(\frac{j-i-1+\operatorname{sgn} i}{2(n-1)}-\frac{1}{2}\right)\left(1-\frac{4}{n}+\frac{2}{n^{2}}\right)^{t} .
$$


For $1 \leq i \leq n$, the probability that $\pi_{-i}^{(t)}>\pi_{i}^{(t)}$ is

$$
\frac{1}{2}-\frac{1}{2}\left(1-\frac{2}{n}\right)^{t}
$$

Proof. Part (a) of the theorem follows from part (b) by the simple observation that $\sum_{j>|i|}(j-i)=2 n\left(n^{2}-1\right) / 3$.

It remains to prove part (b). For every $i, j \in[-n, n] \backslash\{0\}$ and $t \geq 0$, let

$$
p_{i, j}^{(t)}:=\operatorname{Prob}\left(\pi_{i}^{(t)}<\pi_{j}^{(t)}\right) \quad \text { and } \quad u_{i, j}^{(t)}:=n^{2 t} p_{i, j}^{(t)}
$$

The following recurrence relation holds:

$$
\begin{aligned}
u_{i, j}^{(t+1)}= & n^{2} u_{i, j}^{(t)}+\left(u_{j, i}^{(t)}-u_{i, j}^{(t)}\right)+\left(u_{-j,-i}^{(t)}-u_{i, j}^{(t)}\right) \\
& +\sum_{\left|i^{\prime}\right| \neq|j|}\left(u_{i^{\prime}, j}^{(t)}-u_{i, j}^{(t)}\right)+\sum_{\left|j^{\prime}\right| \neq|i|}\left(u_{i, j^{\prime}}^{(t)}-u_{i, j}^{(t)}\right) \quad \text { if }|j| \neq|i|, \text { and } \\
u_{-i, i}^{(t+1)}= & n^{2} u_{-i, i}^{(t)}+\left(u_{i,-i}^{(t)}-u_{-i, i}^{(t)}\right)+\sum_{\left|i^{\prime}\right| \neq|i|}\left(u_{-i^{\prime}, i^{\prime}}^{(t)}-u_{-i, i}^{(t)}\right) .
\end{aligned}
$$

We also have the symmetry property $u_{-j,-i}^{(t)}=u_{i, j}^{(t)}$.

Now, define $v_{i, j}^{(t)}:=u_{i, j}^{(t)}-u_{j, i}^{(t)}$ for $i, j \in[-n, n] \backslash\{0\}$. Clearly, $v$ inherits the symmetry property from $u$ and also has the antisymmetry property $v_{j, i}^{(t)}=-v_{i, j}^{(t)}$. The above recurrence for $u$ is valid also for $v$, and using the symmetry and antisymmetry properties it can be written as

$$
\begin{aligned}
v_{i, j}^{(t+1)} & =\left(n^{2}-4 n+2\right) v_{i, j}^{(t)}+\sum_{\left|i^{\prime}\right| \neq|j|} v_{i^{\prime}, j}^{(t)}+\sum_{\left|j^{\prime}\right| \neq|i|} v_{i, j^{\prime}}^{(t)} \quad \text { if }|j| \neq|i|, \text { and } \\
v_{-i, i}^{(t+1)} & =n(n-2) v_{-i, i}^{(t)} .
\end{aligned}
$$

Clearly, this implies that $v_{i,-i}^{(t)}=\left(n^{2}-2 n\right)^{t}$ for $1 \leq i \leq n$, and if $j>|i|$, Lemma 5.4 yields

$$
v_{i, j}^{(t)}=\frac{j-i-1+\operatorname{sgn} i}{n-1}\left(n^{2}-2 n\right)^{t}+\left(1-\frac{j-i-1+\operatorname{sgn} i}{n-1}\right)\left(n^{2}-4 n+2\right)^{t} .
$$

After dividing this by $n^{2 t}$ we obtain

$$
\begin{aligned}
& 1-2 p_{j, i}^{t}=p_{i, j}^{t}-p_{j, i}^{t} \\
& =\frac{j-i-1+\operatorname{sgn} i}{n-1}\left(1-\frac{2}{n}\right)^{t}+\left(1-\frac{j-i-1+\operatorname{sgn} i}{n-1}\right)\left(1-\frac{4}{n}+\frac{2}{n^{2}}\right)^{t}
\end{aligned}
$$

if $j>|i|$ and

$$
1-2 p_{-i, i}^{t}=\left(1-\frac{2}{n}\right)^{t}
$$

for $1 \leq i \leq n$. This proves part (b) of the theorem.

5.4. The groups of type D. Recall that $D_{n}$ is isomorphic to the subgroup of permutations $\pi$ of the set $[-n, n] \backslash\{0\}$ such that $\pi_{-i}=-\pi_{i}$ for all $1 \leq i \leq n$ and there is an even number of $i \in\{1,2, \ldots, n\}$ such that $\pi_{i}<0$ (again see e.g. [1, Ch. 8]). In this representation, the set of reflections is

$$
\{(i, j)(-i,-j): 1 \leq|i|<j \leq n\}
$$


in cycle notation. The length of $\pi$ equals its number of $D$-inversions, which are pairs $(i, j)$ with $i, j \in[-n, n] \backslash\{0\}$ and $j>|i|$ such that $\pi_{i}>\pi_{j}$.

Theorem 5.6. For $n \geq 1$ and $t \geq 0$, let $\pi^{(t)}$ be a product of $t$ random reflections in $D_{n}$. Then the following hold.

(a) The expected length of $\pi^{(t)}$ is

$$
E_{T, \ell}^{D_{n}}(t)=\frac{n(n-1)}{2}-\frac{n(2 n-1)}{6}\left(1-\frac{2}{n}\right)^{t}-\frac{n(n-2)}{6}\left(1-\frac{4}{n}\right)^{t} .
$$

(b) If $i, j \in[-n, n] \backslash\{0\}$ and $j>|i|$, the probability that $\pi_{i}^{(t)}>\pi_{j}^{(t)}$ is

$$
\frac{1}{2}-\frac{j-i-1+\operatorname{sgn} i}{2(n-1)}\left(1-\frac{2}{n}\right)^{t}+\left(\frac{j-i-1+\operatorname{sgn} i}{2(n-1)}-\frac{1}{2}\right)\left(1-\frac{4}{n}\right)^{t} .
$$

Proof. Part (a) of the theorem follows from part (b) by the simple observation that $\sum_{j>|i|}(j-i)=2 n\left(n^{2}-1\right) / 3$.

It remains to prove part (b). For every $t \geq 0$ and $(i, j) \in \mathcal{I}_{n}$, let

$$
p_{i, j}^{(t)}:=\operatorname{Prob}\left(\pi_{i}^{(t)}<\pi_{j}^{(t)}\right) \quad \text { and } \quad u_{i, j}^{(t)}:=\left(n^{2}-n\right)^{t} p_{i, j}^{(t)} .
$$

The following recurrence relation holds:

$$
\begin{aligned}
u_{i, j}^{(t+1)}= & \left(n^{2}-n\right) u_{i, j}^{(t)}+\left(u_{j, i}^{(t)}-u_{i, j}^{(t)}\right)+\left(u_{-j,-i}^{(t)}-u_{i, j}^{(t)}\right) \\
& +\sum_{\left|i^{\prime}\right| \neq|i|,|j|}\left(u_{i^{\prime}, j}^{(t)}-u_{i, j}^{(t)}\right)+\sum_{\left|j^{\prime}\right| \neq|i|,|j|}\left(u_{i, j^{\prime}}^{(t)}-u_{i, j}^{(t)}\right) .
\end{aligned}
$$

We also have the symmetry property $u_{-j,-i}^{(t)}=u_{i, j}^{(t)}$.

Now, define $v_{i, j}^{(t)}:=u_{i, j}^{(t)}-u_{j, i}^{(t)}$ for $(i, j) \in \mathcal{I}$. Clearly, $v^{(t)}$ inherits the symmetry property from $u^{(t)}$ and also has the antisymmetry property $v_{j, i}^{(t)}=-v_{i, j}^{(t)}$. The above recurrence for $u$ is valid also for $v$, and using the symmetry and antisymmetry properties it can be written as

$$
v_{i, j}^{(t+1)}=\left(n^{2}-5 n+4\right) v_{i, j}^{(t)}+\sum_{\left|i^{\prime}\right| \neq|j|} v_{i^{\prime}, j}^{(t)}+\sum_{\left|j^{\prime}\right| \neq|i|} v_{i, j^{\prime}}^{(t)} .
$$

By Lemma 5.4 we obtain, for $j>|i|$,

$$
v_{i, j}^{(t)}=\frac{j-i-1+\operatorname{sgn} i}{n-1}\left(n^{2}-3 n+2\right)^{t}+\left(1-\frac{j-i-1+\operatorname{sgn} i}{n-1}\right)\left(n^{2}-5 n+4\right)^{t} .
$$

Dividing by $\left(n^{2}-n\right)^{t}$ yields

$$
\begin{aligned}
& 1-2 p_{j, i}^{t}=p_{i, j}^{t}-p_{j, i}^{t} \\
& =\frac{j-i-1+\operatorname{sgn} i}{n-1}\left(1-\frac{2}{n}\right)^{t}+\left(1-\frac{j-i-1+\operatorname{sgn} i}{n-1}\right)\left(1-\frac{4}{n}\right)^{t}
\end{aligned}
$$

if $j>|i|$. This proves part (b) of the theorem. 


\section{Conclusion And ideas For Future ReSEARCH}

We can sum up the current state of knowledge by the following table, listing the groups $W$ for which we have exact expressions for $E_{R, \varphi}^{W}(t)$. (Theorem 3.4 is not included in this list, since we do not know of any generalization of the fixed-point distance to other Coxeter groups than the symmetric group.)

\begin{tabular}{l||c|c} 
& $R=$ simple reflections & $R=$ all reflections \\
\hline \hline$\varphi=$ length & $A_{n}, I_{2}(m), I_{2}(\infty)$ & $A_{n}, B_{n}, D_{n}, I_{2}(m)$ \\
\hline$\varphi=$ absolute length & $I_{2}(m), I_{2}(\infty)$ & $A_{n}, B_{n}, I_{2}(m), G(r, 1, n)$
\end{tabular}

Perhaps the two most striking things with this table are that $B_{n}$ is missing from the upper left square and that not even $A_{n}$ appears in the lower left square!

As Troili has pointed out [8], the problem of computing $E_{S, \ell}^{B_{n}}(t)$ can be reduced to a heat flow process similar to that of the symmetric group, but it is not clear how to analyze it further.

Computing $E_{S, \ell^{\prime}}^{A_{n}}(t)$ is equivalent to computing the expected number of cycles (in the cycle representation of the permutation) after applying $t$ random adjacent transpositions to the identity permutation. For now, we have no idea how to attack this natural problem. Note that it is not enough to keep track of the conjugacy class of the permutation as Eriksen and Hultman did in the proof of Theorem 3.3.

It is also worth noting that $E_{S, \ell}^{W}(t)$ and $E_{S, \ell^{\prime}}^{W}(t)$ are well-defined for any finitely generated Coxeter system. (Indeed, Troili made an effort to compute $E_{S, \ell}^{\tilde{A}_{n}}(t)$ in $[8]$, but unfortunately with an erroneous heat flow process.)

In addition to the length and the absolute length, there is at least one more "length" function that comes naturally with any Coxeter system $(W, S)$, namely the descent number defined by

$$
d(w)=\#\{s \in S: \ell(w s)<\ell(w)\} .
$$

(In fact, the ordinary length function is also a kind of descent number by the equality $\ell(w)=\#\{t \in T: \ell(w t)<\ell(w)\}$; see e.g. [1, Corollary 1.4.5].) What are $E_{S, d}^{W}(t)$ and $E_{T, d}^{W}(t)$ for a (in the second case, finite) Coxeter system $(W, S)$ ?

\section{ACKNOWLEDGEMENTS}

This work was performed at KTH in Stockholm and was supported by a grant from the Swedish Research Council (621-2009-6090).

\section{REFERENCES}

1. Anders Björner and Francesco Brenti, Combinatorics of Coxeter groups, Springer, 2005. MR2133266 (2006d:05001)

2. Mireille Bousquet-Mélou, The expected number of inversions after $n$ adjacent transpositions, Discrete Math. Theor. Comput. Sci. 12 (2010), 65-88. MR2676666

3. Niklas Eriksen, Expected number of inversions after a sequence of random adjacent transpositions - an exact expression, Discrete Math. 298 (2005), 155-168. MR2163446 (2006b:05008)

4. Niklas Eriksen and Axel Hultman, Estimating the expected reversal distance after a fixed number of reversals, Adv. in Appl. Math. 32 (2004), 439-453. MR2041958 (2004m:92018)

5. _ Expected reflection distance in $g(r, 1, n)$ after a fixed number of reflections, Ann. Comb. 9 (2005), 21-33. MR2135773 (2005m:05228) 
6. Henrik Eriksson, Kimmo Eriksson, and Jonas Sjöstrand, Expected number of inversions after a sequence of random adjacent transpositions, Proceedings of Formal Power Series and Algebraic Combinatorics (D. Krob, A. A. Mikhalev, and A. V. Mikhalev, eds.), Springer, Berlin, 2000, pp. 677-685. MR.1798262 (2001i:05018)

7. Andreas Jönsson, Evolutionary fixed point distance problems, master's thesis, University of Gothenburg, 2009.

8. Emma Troili, Förväntade avstånd $i$ Coxetergrupper [Expected distances in Coxeter groups], master's thesis, KTH, 2002, in Swedish.

Department of Mathematics, Royal Institute of Technology, SE-100 44 Stockholm, SWEDEN

E-mail address: jonass@kth.se 